\title{
Childhood developmental disorders
}
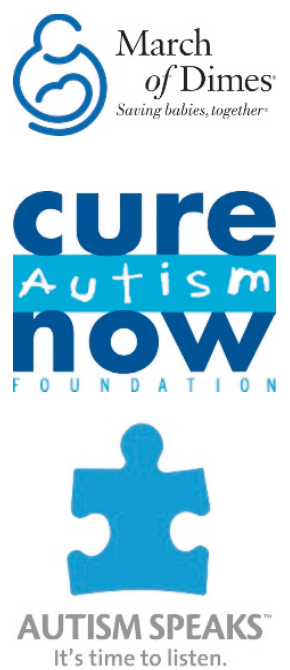

embryonic development. Some researchers have suggested that environmental factors may be involved in language disorders as well. The genetic causes of many mental retardation syndromes, in contrast, are attributable to a specific locus encompassing one or several genes.

Symptoms are usually first visible in childhood, though their effects persist throughout life. As yet, there are no definitive treatments for most of these disorders, only palliative treatments that help those affected to cope with their symptoms. Affected children and their families have to deal with these disorders throughout their lives. Because many of the symptoms manifest in educational settings, schools also need to take into account the needs of these children.

The study of childhood developmental disorders has been very active in the last thirty years. Researchers have usually examined these disorders independently, but it is becoming increasingly clear that there may be commonalities among them. Studying abnormal development may yield information about normal developmental processes as well. In this Focus, we present four Perspective articles that explore this overlap between different developmental disorders, and normal and abnormal development.

Perhaps no other developmental disorder has received as much attention as dyslexia, which is not surprising considering its impact on the educational progress of otherwise bright children. However, there is surprisingly little consensus on its etiology and treatment. One promising line of inquiry is the genetic basis of dyslexia, but although several genes contribute to the disorder, it is not clear how they cause the behavioral deficit. Albert Galaburda and colleagues propose a speculative pathway from these genes to specific neural circuits to behavior. This Perspective underlines the need for research on very early behavior in those children at risk for dyslexia.

Another developmental disorder that has been a major focus of attention is autism, which, though less prevalent than dyslexia, can be far more devastating in terms of normal social functioning. Many hypotheses have been proposed to explain the central deficits in autism, but no proposal has successfully accounted for all of the symptoms. In their Perspective, Francesca Happé, Angelica Ronald and Robert Plomin argue that no single defect is likely to explain the core symptoms because autism consists of a constellation of traits found (in less extreme form) in the normal population as well and inherited largely independently of each other.

Gary Marcus and Hugh Rabagliati also argue in their Perspective that the gap between normal and abnormal development may not be as wide as previously assumed. Many of the most common developmental disorders (including SLI, dyslexia and autism) involve major disturbances in language. This Perspective proposes that studying these disorders could provide part of the answer to a question of great interest to linguists and neuroscientists alike: how did language evolve in humans? This line of inquiry has often been hampered by the lack of hard data (language does not fossilize), but in combination with studies on language-like processing in nonhuman primates, research on these developmental disorders could help test many of the hypotheses in this field.

In the final Perspective, Matthew Belmonte and Thomas Bourgeron show how an unconventional approach can illuminate a pressing research question. They compare autism and fragile $\mathrm{X}$ syndrome, two developmental disorders that seem at first sight to have very different etiologies and symptoms. However, this comparison provides clues to the genetic and neural networks involved in both disorders, and shows that developmental disorders may have more in common than previously suspected.

We are grateful to the March of Dimes, Cure Autism Now and Autism Speaks for their generous financial support of this focus issue, which has allowed us to make its content freely available on the web for three months at http://www.nature.com/neuro/focus/childhood/index.html. Apart from the sponsor's foreword, the editorial team of Nature Neuroscience is entirely responsible for the content of the Focus issue. We hope that our readers will find this collection of articles useful and enlightening and that they will spur future research in this important field.

\section{Charvy Narain \\ Associate Editor}

